\title{
Online Credit System using Face Recognition
}

\author{
Vaibhav Ambasta \\ Student, Department of Computer \\ Science and Engineering \\ B.M.S. College of Engineering \\ Bangalore, India
}

\author{
Rakshith S. M. \\ Student, Department of Computer \\ Science and Engineering \\ B.M.S. College of Engineering \\ Bangalore, India
}

\author{
Umadevi V. \\ Associate Professor, Department \\ of Computer Science and \\ Engineering \\ B.M.S. College of Engineering \\ Bangalore, India
}

\author{
Tenzin Kunsang \\ Student, Department of Computer \\ Science and Engineering \\ B.M.S. College of Engineering \\ Bangalore, India
}

\begin{abstract}
This paper proposes a credit system employing face recognition that will enable payment transaction process faster than existing systems. Supermarkets are often slow when processing payments. For example, the customer will have to provide his/her card and then enter the pin number details to the card swiping machine. The work described in this paper aims to simplify the payment processing interface, with minimum user interference, and ensuring a fast payment checkout. Deep learning techniques have been deployed in the proposed architecture, largely composed of convolutional neural networks. All that is required to process a payment transaction is the customer's face image, and nothing else. The customer's face, once recognized, will enable the system to fetch his bank account details and allow the transaction to proceed by debiting the required amount from his/her bank account linked to the payment system. The face recognition model described in this paper has a $100 \%$ accuracy in predicting the correct output. The focus of this work is to recognize customer's face for online payment.
\end{abstract}

\section{General Terms}

Face Recognition, Deep learning, Model, Dataset, Online Payment System, Cost Function, Neural Networks

\section{Keywords}

Anchor image, Convolutional neural networks, Face recognition, Facenet model, Inception networks, MTCNN model, Triplet loss function

\section{INTRODUCTION}

Computer vision technologies have taken the world by storm. They have been versatile and have been used in areas likemedical diagnosis, satellite imaging, face recognition tasks, etc. There have been regular advancements in this -field and the better results have led to their applications across a diverse set of fields. The proposed online credit system described in this paper can be used by market vendors, as a payment checkout interface. This system needs minimum customer involvement and just requires the face image of the customer. After the customer's face is recognized, the bill amount gets automatically deducted from the customer's bank account that is linked to the payment system. The system's main component is face recognition. In this paper, details as to how the task of implementing the online credit system using face recognition is achieved have been described. An image is first passed to our face recognition model. The model outputs a 128 length vector encoding. This encoding is then compared to the embedding of each person's image stored in the database.

An overview of the rest of the paper is as follows: Section $2-$ Review of the literature relating to this work, Section 3 Describes the proposed architecture design of the system, Section 4 - Discusses on different methods that were used to train our model, Section 5 - Deals with the dataset that was used to train our model, and finally, Section 6 - Results of the proposed our model will be presented..

\section{RELATED WORKS}

There has been a lot of work on face recognition related tasks. Some of the most popular works are-

DeepFace model[1] by Facebook, Facenet model [2] by Google, MTCNN model (Multi-task Cascaded Convolutional Network) [3], etc. These models have high accuracy in recognizing face image, the DeepFace model [1] has $97.35 \%$ on the LFW dataset and Facenet model [2] has up to 95.12\% on the YouTube Faces Database. These models have many common components in their architecture. These models are largely composed of deep Convolutional Neural Networks with varying sizes of filters. A repetitive architecture is observed in these models[2], there have been variations with the inclusion of Inception Networks [2]. The MTCNN model[3] focuses on extracting the facial region in an image. A multi-task cascaded CNN framework is employed for joint face detection and alignment. Training the model is based on 3 stages- face classification, bounding box regression, and facial landmark localization. Learning was based on backpropagation. The Facenet model [2] consists of deep convolutional networks with inception networks, which help in cases of different feature sizes. The learning phase is based on a triplet loss function, which involves the anchor, positive and negative images. The model tries to maximize the difference between the anchor image and the negative image, while reducing the difference between the anchor image and the positive image. It achieves a classification accuracy of $98.87 \%$ on the LFW dataset. DeepFace model [1] highly stresses on face alignment tasks, based on a Siamese Network model. A multi-class network is trained to perform face recognition task on over 4000 identities. Weights are learned 
based on the standard back propagation technique. This model is efficient in terms of computational complexity- it runs at 0.33 seconds per image, which involves image decoding, face detection and alignment, feed forward network and the final classification output.

The FaceNet model is shown to outperform several other state of the art models. The results have been clearly portrayed by the deformable FaceNetmodel[4], which gives a higher accuracy rate compared to the CosFace model and the ResNet 152 model. Face Recognition based Surveillance System Using FaceNet and MTCNN on Jetson TX2 [5] was successfully implemented with multiple cameras. This standalone system detects the person using their face image and an embedding being created was successfully detected with an accuracy of $97 \%$.

\section{PROPOSED DESIGN OF THE SYSTEM}

The problem statement can be well summarized by the diagram shown in Figure 1.In a broader sense, the high-level design of the entire work has three main modules:

\section{MTCNN model}

2. Face Recognition model (FaceNet)

3. Comparison of image embedding

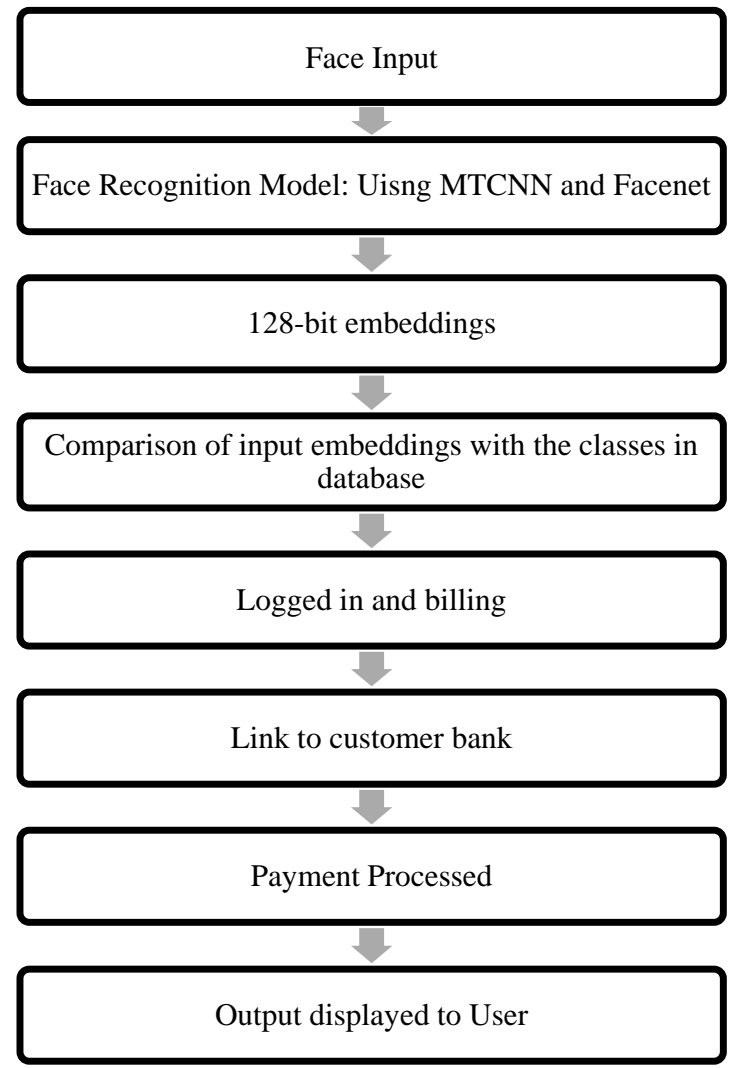

Fig 1: Face Recognition using FaceNet Model and MTCNN model

The MTCNN model is used to provide the facial region of any given input image. The output is then passed on to the face recognition model. A third party source code implementation [6] of the Facenet model [2] has been used. The FaceNet model outputs a 128 length vector embedding. The final task involves the comparison of this embedding to the embedding of images of different people stored in the database. The proposed credit system employs a face recognition model based on the Facenet model [2] by Google. The model was trained on the CASIA WebFace dataset [7].The model's architecture is based on convolutional neural networks, with inception networks also being a part of it in some layers. The extensive application of the inception modules[8] is a key component in the model. Inception modules make use of filters with multiple sizes that operate on same level. This is in contrast to plain convolutional networks, where all filters operating on any level have the same dimensions. The advantage of having filters of different sizes operate on the same level is that the model is better at identifying salient features in the image, which may have large variations in size. Inception networks have been the go to solution in instances where it has been hard to choose the right filter size.

The detailed architecture for the MTCNN model and FaceNet model are shown in Table I and Table II respectively. The model take input as an image and the output generated by the model is a 128 dimensional vector. Euclidean distance is used to check for similarity among the vectors. The database has embedding of face image of different people. When a new embedding is received, we classify the embedding as belonging to the person with whom the Euclidean distance is the least, and is below a certain threshold. If there is no person for whom the Euclidean distance is below the threshold, the person's image will not be validated.

Table 1. Architecture of Mtcnn Model

\begin{tabular}{|c|c|c|c|c|c|}
\hline layer & kernel & filters & input & output & Parameters \\
\hline Conv1 & $3 \times 3$ & 24 & $32 \times 32 \times 3$ & $30 \times 30 \times 24$ & 648 \\
Prelu1 & & & $30 \times 30 \times 24$ & $30 \times 30 \times 24$ & 24 \\
\hline Conv2 & $4 \times 4$ & 24 & $30 \times 03 \times 24$ & $14 \times 14 \times 24$ & 9216 \\
Prelu2 & & & $14 \times 14 \times 24$ & $14 \times 14 \times 24$ & 24 \\
\hline Conv3 & $4 \times 4$ & 32 & $14 \times 14 \times 24$ & $11 \times 11 \times 32$ & 12288 \\
Prelu3 & & & $11 \times 11 \times 32$ & $11 \times 11 \times 32$ & 32 \\
\hline $\begin{array}{c}\text { Conv4 } \\
\text { Prelu4 }\end{array}$ & $4 \times 4$ & 48 & $11 \times 11 \times 32$ & $8 \times 8 \times 48$ & 24576 \\
\hline Conv5 & $4 \times 4$ & 32 & $8 \times 8 \times 48$ & $5 \times 5 \times 32$ & 24576 \\
Prelu5 & & & $5 \times 5 \times 32$ & $5 \times 5 \times 32$ & 32 \\
\hline $\begin{array}{c}\text { Conv6 } \\
\text { Prelu6 }\end{array}$ & $3 \times 3$ & 16 & $5 \times 5 \times 32$ & $3 \times 3 \times 16$ & 4608 \\
\hline $\begin{array}{l}\text { Conv7 } \\
\text { Prelu7 }\end{array}$ & $3 \times 3$ & 2 & $3 \times 3 \times 16$ & $1 \times 1 \times 2$ & 288 \\
\hline
\end{tabular}

\section{METHODOLOGY}

There are several methods that are employed to train the face recognition model. The cost function (error loss function) that is used is the triplet loss function [2]. Based on this loss function, the filter weights in the model are changed. Backpropagation is used for the purpose of changing the weight values. The final step is to classify a given image, to one of the similar class among several classes that are present in the database. The concept of Euclidean distance helps with this task. 
Table 2. Architecture of FaceNet model by Google

\begin{tabular}{|c|c|}
\hline $\begin{array}{c}\text { Number of kernels/layer } \\
\text { type }\end{array}$ & Kernel size \\
\hline 32 & $3 * 3$ \\
\hline 32 & $3 * 3$ \\
\hline 64 & $3 * 3$ \\
\hline Max-pooling & $3 * 3$ \\
\hline 86 & $1 * 1$ \\
\hline 192 & $3 * 3$ \\
\hline 256 & $3 * 3$ \\
\hline Inception-net (depth=5) & $1 * 1(96), 3 * 3(96)$ \\
\hline Inception-net & $1 * 1(192), 3 * 3(578)$ \\
\hline Inception-net (depth=10) & $1 * 1(256), 1 * 7(128), 7 * 1(128)$ \\
\hline Inception-net & $1 * 1(768), 3 * 3(1154)$ \\
\hline Inception-net (depth=6) & $1 * 1(384), 1 * 3(192), 3 * 1(192)$ \\
\hline Average pooling & $3 * 3$ \\
\hline Fully-connected & Output-128*1 \\
\hline Fully-connected & Output-128*1 \\
\hline
\end{tabular}

The following steps have been used:

\subsection{Facial Region Extraction}

The first step before training is to convert the image to a form that includes only the facial region. Thus, for all images in the data set, there will be a new set of images, which include only the facial region. These new sets of images would be used for training the model.

The process of facial region extraction is done using the MTCNN [3] framework. This model was developed to focus on areas like face classification, bounding box regression, and facial landmark localization. All images, after having been fed through the MTCNN model, are resized to $160 * 160 * 3$, which will be the input to the face recognition model. The MTCNN framework [3] has outperformed other state of the art model on several challenging benchmarks while keeping real-time performance. Therefore, using it would add robustness to the face recognition model.

\subsection{Triplet Loss Function}

The learning phase in the face recognition model involves the use of the triplet loss function [2]. This function aims to ensure that an anchor image of a person is closer to the positive images and farther away from negative images.

Thus, if

- $\alpha$ denotes the margin that is enforced between positive and negative pairs,

- $x^{a}$ denote the anchor image,

- $x^{p}$ denote the positive image,

- $x^{n}$ denote the negative image,

- $\quad \forall$ denotes for all.

Then the following equation sums up the requirement:

$$
\left(x^{a}-x^{p}\right)^{2}+\alpha<\left(x^{a}-x^{n}\right)^{2} \forall\left(x^{a}, x^{p}, x^{n}\right)-
$$

When training, the triplets were selected that violate the triplet constraint, resulting in faster learning of the system. This is the main loss function of the face-recognition model. The triplet loss function is summarized by the Figure 2, which shows that the function learns to minimize distance between positive and anchor image, and tries to maximize the distance between the anchor and negative images.

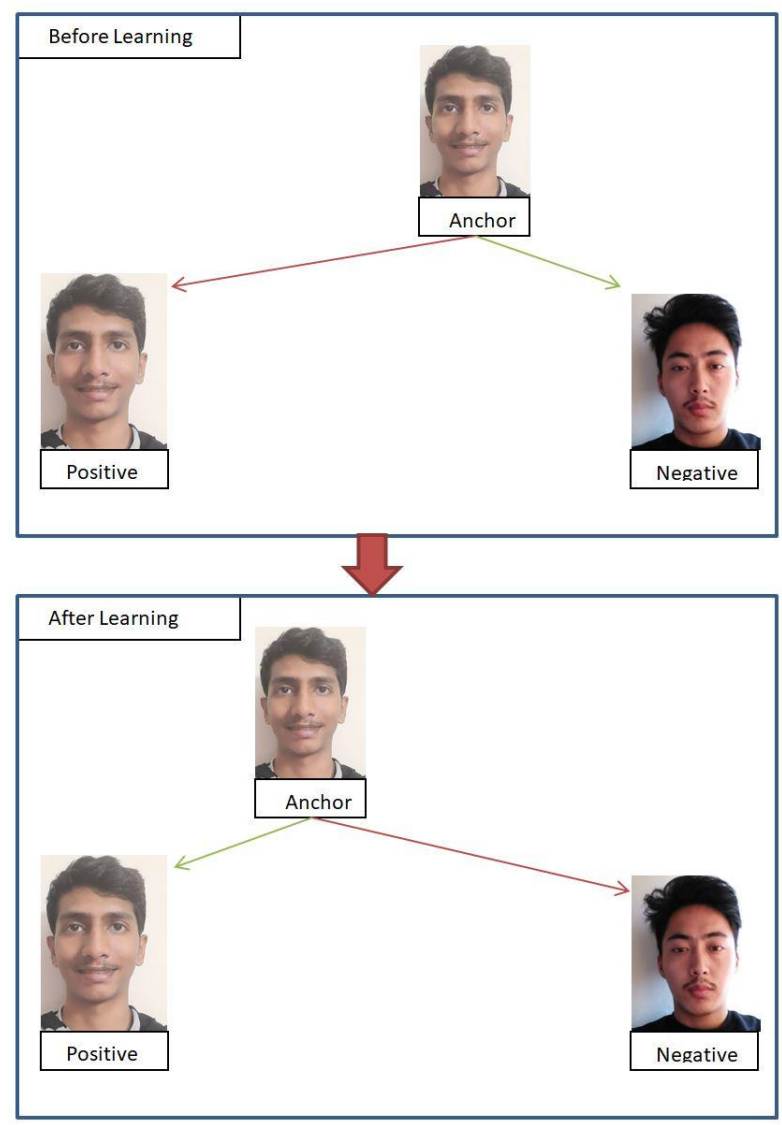

Fig 2: Application of Triplet Loss Function to recognise simliar user's face and comparison of results before and after learning both columns

\subsection{Back-propagation}

The triplet loss function is used as the cost function. The method employed by the model to learn the weights was standard back-propagation, which uses gradient descent. In this case, the filter parameters are randomly initialized at the beginning. They weights are modified, based on the partial derivative of the cost function with respect to the filter parameters. This process is done for all the parameters and several iterations may be required in order to achieve a high degree of performance.

\subsection{Comparison of Image Embedding}

The final stage in the model is to classify the new test image as belonging to a particular class. Euclidean distance is used in the model to determine which class a given embedding belongs to. The model's database has an embedding stored for each person. When a new embedding is received, it is compared to every other embedding in the database. A certain threshold used in determining the output class, failing which the input image will not be validated. 


\section{DATASETS}

The dataset used to train the model was the CASIA Web Face [7]. This dataset consists of about $500 \mathrm{~K}$ images spread across 10,575 subjects.

\section{RESULT}

The model is deployed on the server using Flask framework. The server has provisions for live detection, as well as to add new users and predict the class for a new image. The model's final classification task achieves an accuracy of $100 \%$. We have deployed one-shot learning [5] wherein only one training image per customer is needed to train the model. One-shot learning proves beneficial on a large scale, not putting load on the system and facilitating fast response time as well.

Table 3 shows the recognition of an image with high accuracy, that was already trained, and Figure 3, shows that system cannot recognize an image, which is not trained.

The different models and their results can be compared, as shown in Table IV. The CASIA Webface Dataset was used as the training set for the model, and for testing the performance a different dataset, the Youtube Faces DB[9] was used.

Table 3. High Accuracy is achieved by Successfully detecting the Customer's Identities

\begin{tabular}{|c|c|c|c|}
\hline Input image & $\begin{array}{c}\text { Predicted } \\
\text { Output }\end{array}$ & $\begin{array}{c}\text { Actual } \\
\text { Output }\end{array}$ & Accuracy \\
\hline & Rakshith & Rakshith & $100 \%$ \\
\hline & Tenzin & Tenzin & $100 \%$ \\
\hline & & & \\
\hline
\end{tabular}

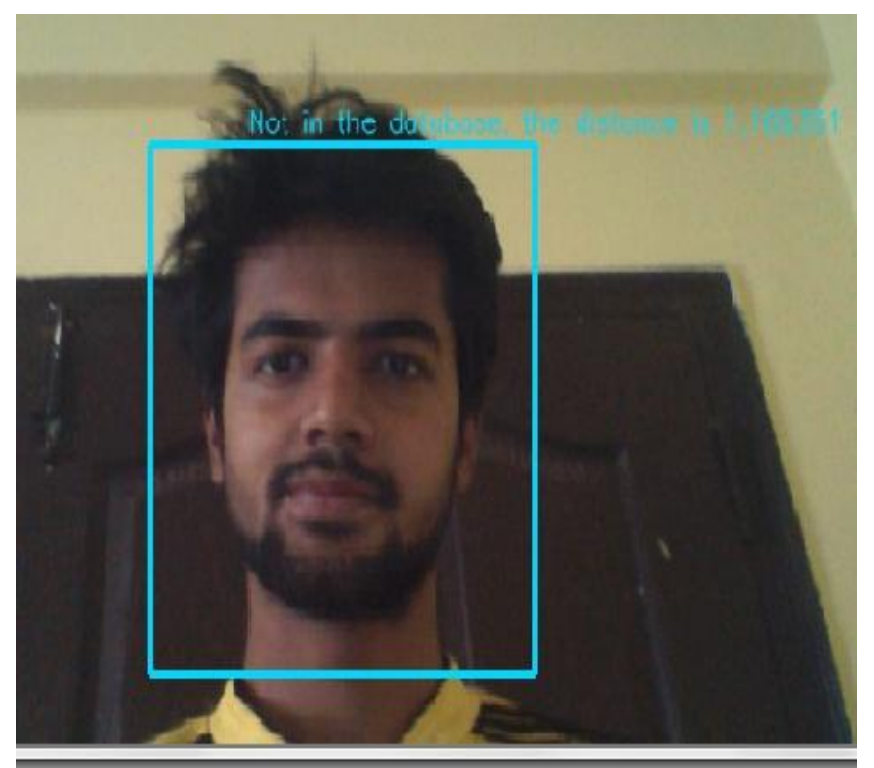

Fig. 3:System cannot recognize an image, which is not trained.
Table 4 Comparision of Facenet with other models

\begin{tabular}{|c|c|c|c|}
\hline Dataset & \multicolumn{3}{|c|}{ Percentage of Accuracy } \\
& $\begin{array}{c}\text { Facenet } \\
\text { Model[2] }\end{array}$ & $\begin{array}{c}\text { Deepface } \\
\text { Model[1] }\end{array}$ & $\begin{array}{c}\text { deepID2 } \\
\text { Model[10] }\end{array}$ \\
\hline $\begin{array}{c}\text { Youtube } \\
\text { FacesDB[9] }\end{array}$ & $95.12 \%$ & $91.4 \%$ & $93.2 \%$ \\
& & & \\
\hline
\end{tabular}

\section{CONCLUSION AND FUTURE SCOPE}

The proposed face recognition model in this work has nearly $100 \%$ accuracy. The inclusion of MTCNN framework allowed the model to focus more on the subtle features in people's faces, and inception blocks allowed to capture variance in feature sizes. This could be a great replacement for card payment by customers and vendors, takes less time and also avoids the possibility of forgery. However, the model lacks liveliness detection, and in some cases can be fooled by being shown an image of a person. To counter this in future, the model could be trained so that it is able to detect live frames.

Deploying one-shot learning on a large scale is challenging, as there is only one image per customer. In such a scenario, a Siamese network that is trained on thousands of images would be beneficial. Moreover, tests should be conducted over images captured by different cameras.

\section{REFERENCES}

[1] Taigman, Yaniv, Ming Yang, Marc'Aurelio Ranzato, and Lior Wolf. "Deepface: Closing the gap to human-level performance in face verification." InProceedings of the IEEE conference on computer vision and pattern recognition, pp. 1701-1708. 2014 Location: Columbus, Ohio (USA) Date: June 2014.

[2] Schroff, Florian, Dmitry Kalenichenko, and James Philbin. "Facenet: A unified embedding for face recognition and clustering." Proceedings of the IEEE conference on computer vision and pattern recognition. 2015. Location: Boston, Massachusetts (USA) Date: June 2015 Version: $3^{\text {rd }}$ version

[3] Xiang, Jia, and Gengming Zhu. "Joint Face Detection and Facial Expression Recognition with MTCNN."2017 4th International Conference on Information Science and Control Engineering (ICISCE). IEE 2017. Location: Changsha, China Date: July 2017

[4] He, Mingjie, Jie Zhang, Shiguang Shan, Meina Kan, and Xilin Chen. "Deformable Face Net: Learning Pose Invariant Feature with Pose Aware Feature Alignment for Face Recognition." In 2019 14th IEEE International Conference on Automatic Face \& Gesture Recognition (FG 2019), pp. 1-8. IEEE, 2019. Location: Lille, France Date: May 2019

[5] Jose, Edwin, M. Greeshma, Mithun Haridas TP, and M. H. Supriya. "Face recognition based surveillance system using facenet and mtcnn on jetson tx2." In 2019 5th International Conference on Advanced Computing \& Communication Systems (ICACCS), pp. 608-613. IEEE, 
2019. Location: Coimbatore, India Date: March 2019

[6] Github, URL: https://github.com/davidsandberg/facenet [Last accessed: Apr 2018]

[7] D. Yi, Z. Lei, S. Liao, and S. Z. Li, "Learning face representation from scratch," 2014, arXiv:1411.7923. [Online]. Available: http://arxiv.org/abs/1411.7923 Date: November 2014 Version: $1^{\text {st }}$ Version

[8] Szegedy, Christian, Wei Liu, Yangqing Jia, Pierre Sermanet, Scott Reed, Dragomir Anguelov, Dumitru Erhan, Vincent Vanhoucke, and Andrew Rabinovich. "Going deeper with convolutions." In Proceedings of the
IEEE conference on computer vision and pattern recognition, pp. 1-9. 2015. Location: Boston, Massachusetts (USA) Date: June 2015

[9] L. Wolf, T. Hassner, and I. Maoz. Face recognition in unconstrained videos with matched background similarity. In IEEE Conf. on CVPR, 2011. 5 Location: Colorado Spring, Colorado (USA) Date: June 2011

[10] Sun, Y., Chen, Y., Wang, X., \& Tang, X. (2014). Deep learning face representation by joint identificationverification. In Advances in neural information processing systems (pp. 1988-1996). Date: June 2014 Version: $1^{\text {st }}$ 